\title{
Clonación, expresión y evaluación inmunológica de la proteína Omp31 de Brucella melitensis y evaluación de su posible uso para el diagnóstico en brucelosis bovina
}

\author{
Cloning, expression and immunological eValuation of the OMp31 PROTEIN OF \\ Brucella melitensis AND EVALUATION OF ITS POSSIBLE USE FOR THE DIAGNOSIS \\ IN BOVINE BRUCELLOSIS
}

\author{
Jose-David Rosales ${ }^{1,5}$, Annie Castillo Ochoa ${ }^{1}$, Armando Reyna-Bello ${ }^{2}$, \\ Ana Teresa Serrano ${ }^{3}$, Rodolfo Fernández-Gomez ${ }^{4}$
}

\section{Resumen}

\begin{abstract}
El diagnóstico serológico de la brucelosis se lleva a cabo por la detección de anticuerpos generados contra el liposacárido «LPS» o extractos bacterianos de células enteras por ELISA o pruebas de aglutinación. El objetivo del trabajo fue evaluar una proteína recombinante que pueda ser usada en el diagnóstico serológico de la brucelosis bovina. Se escogió el gen de la Omp31 de Brucella melitensis 16M. El gen fue clonado a partir de ADN de B. melitensis. La expresión de la proteína Omp31r se realizó en un sistema de expresión procariota utilizando el vector pET28a y la purificación por cromatografía de afinidad a metales (IMAC). Un ELISA indirecto fue estandarizado utilizando sueros controles de bovinos positivos y negativos. La proteína Omp31r reaccionó
\end{abstract}

${ }^{1}$ Centro de Inmunoproducción Masiva de Anticuerpos, Fundación Instituto de Estudios Avanzados (IDEA), Caracas, Venezuela

${ }^{2}$ Grupo de Investigación en Sanidad Animal y Humana (GISAH), Carrera Ingeniería en Biotecnología, Departamento de Ciencias de la Vida, Universidad de las Fuerzas Armadas ESPE, Santo Domingo, Ecuador

${ }^{3}$ Facultad de Ciencias Veterinarias, Universidad Central de Venezuela, Maracay, Venezuela

${ }^{4}$ Grupo de Investigación en Sanidad Animal y Humana (GISAH), Carrera Ingeniería en Biotecnología, Departamento de Ciencias de la Vida, Universidad de las Fuerzas Armadas ESPE, Sangolquí, Ecuador; Programa Prometeo, SENESCYT, Biophysic Solutions CA, Caracas, Venezuela

${ }^{5}$ E-mail:jdrr55@yahoo.com

Trabajo financiado por los proyectos: 26193 BID-FONACIT II y Sub-Proyecto $N^{\circ} 12007001425$ Misión Ciencias FONACIT

El trabajo representa parte de la tesis doctoral de José David Rosales en Biotecnología, Mención Animal, de la Escuela Socialista de Agricultura Tropical (ESAT)

Recibido: 28 de noviembre de 2017

Aceptado para publicación: 31 de mayo de 2018 
con los sueros controles positivos y logró discriminarlos frente a los sueros negativos. La sensibilidad y especificidad del ensayo de ELISA fue de $77.2 \%$ y de $90.6 \%$, respectivamente. Este sistema de ELISA indirecto puede ser útil para los diagnósticos rápidos de grandes números de muestras en el diagnóstico de la brucelosis bovina.

Palabras clave: brucelosis; Omp31r; proteína recombinante

\section{AbSTRACT}

The serological diagnosis of brucellosis is carried out by the detection of antibodies generated against the liposaccharide «LPS» or bacterial extracts of whole cells by ELISA or agglutination tests. The objective of this study was to evaluate a recombinant protein that can be used in the serological diagnosis of bovine brucellosis. The Omp31 gene of Brucella melitensis 16M was chosen. The gene was cloned from DNA of B. melitensis; the expression of the Omp31r protein was performed in a prokaryotic expression system using the pET28a vector and purification by metal affinity chromatography (IMAC). An indirect ELISA was standardized using control sera from positive and negative bovines. The Omp31r protein reacted with the positive control sera and succeeded in discriminating against the negative sera. The sensitivity and specificity of the ELISA assay was $77.2 \%$ and $90.6 \%$, respectively. This indirect ELISA system can be useful for the rapid diagnosis of large numbers of samples in the diagnosis of bovine brucellosis.

Key words: brucellosis; Omp31r; recombinant protein

\section{INTRODUCCIÓN}

Brucella abortus es un cocobacilo gran negativo, causante de afecciones crónicas debilitantes en humanos, así como aborto e infertilidad en el ganado y otros animales, dando lugar a serias pérdidas económicas en la ganadería y problemas de salud pública a nivel global. No existe una vacuna eficaz y segura para el humano, por lo que la prevención de la infección en animales es una forma de controlar la brucelosis humana.

Se han desarrollado diferentes enfoques de diagnóstico, principalmente cultivo bacteriano, ensayos serológicos y detección de nivel de ADN. Entre estos, los ensayos serológicos se consideran como el método principal por su sensibilidad diagnóstica, disponibilidad y ventaja económica (Al Dahouk et al., 2003). La detección de anticuerpos contra lipopolisacáridos es el elemento prin- cipal en el serodiagnóstico de la brucelosis; sin embargo, presentan reacción cruzada con bacterias como Escherichia coli O157, Salmonella typhimurium y Vibrio cholerae, así como con Yersinia enterocolitica O:9 con la que tiene la reacción cruzada más prominente debido a la alta similitud estructural del O-polisacárido (OPS) (Bundle et al., 1984; Chenais et al., 2012).

El desarrollo de la inmunoproteómica ha allanado el camino para la identificación de proteínas inmunogénicas de varios patógenos (Tjalsma et al., 2008; Hu et al., 2014; Pellon et al,. 2016; Chavez-Fumagalli et al., 2017). La aplicación de proteínas inmunogénicas de $B$. abortus ha demostrado ser un enfoque eficaz para minimizar las reacciones cruzadas en el diagnóstico de la brucelosis (Zhao et al., 2011). Por lo tanto, varios componentes superficiales o citoplásmicos de Brucella se han usado como marcadores potenciales para el diagnóstico de brucelosis, incluida la 
lumazina sintetasa (Goldbaum et al., 1999), la proteína del sistema de secreción de tipo IV VirB5 (Tan et al., 2012), la proteína de membrana externa Omp28 (Thavaselvam et al., 2010) y la proteína periplásmica inmunogénica Bp26 (Tiwari et al., 2011). La proteína de membrana externa Omp31 ha sido evaluada serológicamente en animales diferentes a bovinos, de allí que resultó interesante que fuera reportada usando sueros de bovinos positivos a Brucella abortus biovar S19 (Connolly et al., 2006), mientras que en otro estudio similar usando la cepa vacunal de Brucella abortus RB51 no la hayan detectado (Kim et al. 2014).

Con base en estos estudios, se ha considerado la clonación del gen de Omp31 para expresar la proteína y evaluar su potencial en el serodiagnóstico de brucelosis en bovinos (Vizcaino et al., 1996; Cassataro et al., 2004; Estein et al., 2004). El diagnóstico de la brucelosis se basa principalmente en la detección de anticuerpos específicos, mediante métodos como Rosa de Bengala (RBT), prueba de aglutinación en suero (TAT), ensayo inmunoabsorbente ligado a enzimas (ELISA), prueba de fijación del complemento (CFT) y prueba de anticuerpos fluorescentes (FTA) (Chenchev et al., 1978; Bricker 2002; Nielsen 2002). El RBT como prueba de detección y el TAT como confirmación se consideran como métodos estándares para el diagnóstico serodiagnóstico de la brucelosis en Venezuela (Lord y Flores, 1983; Lord V y Lord R, 1991; Francisco y Vargas, 2002).

La RBT es una técnica económica y rápida; sin embargo, posee baja especificidad por lo que es fundamental desarrollar nuevas técnicas de diagnóstico de detección rápida y eficaces para el diagnóstico de brucelosis. Este estudio tuvo como objetivo evaluar la técnica de ELISA indirecta utilizando una proteína inmunogénica recombinante para garantizar la especificidad diagnóstica y confirmar el diagnóstico en animales que han sido diagnosticados con brucelosis con técnicas complementarias.

\section{Materiales y Métodos}

\section{Análisis Bioinformático}

Para el diseño de oligos se utilizó el software Oligo Explorer v. 1.4 (http:// www.genelink.com/tools/gl-oe.asp) y la secuencia del gen Omp31de Brucella melitensis con número de acceso GQ184729.1 (https://www.ncbi.nlm.nih.gov/ nuccore/GQ184729.1). Para el alineamiento de las proteínas Omp31 se escogieron tres secuencias de B. abortus: CAJ11595.1 (https://www.ncbi.nlm.nih.gov/protein/ CAJ11595.1), EFH35395.1 (https:// www.ncbi.nlm.nih.gov/protein/EFH35395.1), ACD73018 (https://www.ncbi.nlm.nih.gov/ protein/ACD73018) y una de B. melitensis: GQ184729 (https://www.ncbi.nlm.nih.gov/ nuccore/GQ184729). Estas secuencias se alinearon usando MultAlin (http:// multalin.toulouse.inra.fr/multalin/) y el porcentaje de identidad de aminoácidos se obtuvo usando el software UGENE v. 1.27.0 (http:// ugene.net/).

\section{Cepas Bacterianas}

Una muestra de cepa lisa y virulenta de B. melitensis $16 \mathrm{M}$ fue proporcionada por el Instituto Nacional de Higiene Rafael Rangel (INH «RR»), la cepa atenuada de Brucella abortus biovar 1119 fue cedida por el Instituto Nacional de Investigaciones Agricolas (INIA) y la cepa Escherichia coli BL21(DE3) fue donada por el Centro de Inmunoproducción de Antígenos (CIMA), la cual fue usada para producir las construcciones plasmídicas. La cepa de $E$. coli se cultivó a $37{ }^{\circ} \mathrm{C}$ en caldo Luria-Bertani (LB) o agar suplementado con $30 \mathrm{mg} / \mathrm{ml}$ de kanamicina (Sigma, EEUU).

\section{Preparación del Plásmido}

La secuencia de $723 \mathrm{pb}$ codificante del gen de Omp31 de B. melitensis se amplificó por PCR utilizando el siguiente par de cebadores: Omp31 Forw 5'CCCGGA- 
TCCATGA-AATCCGTAA-TTTTGGC3' (Sitio EcoRI subrayado) y REV 5, CCCGCGGCCGCTTAGAACTTGTAGTTCAGACCG3' (sitio NotI subrayado). La PCR se realizó utilizando los siguientes parámetros: calentamiento a $95{ }^{\circ} \mathrm{C}$ durante $5 \mathrm{~min} ; 32$ ciclos de desnaturalización a $95{ }^{\circ} \mathrm{C}$ durante 1 min, hibridación a $62{ }^{\circ} \mathrm{C}$ durante 1 min y extensión a $72{ }^{\circ} \mathrm{C}$ durante $1 \mathrm{~min}$, y una elongación final a $72{ }^{\circ} \mathrm{C}$ durante $5 \mathrm{~min}$. El ADN amplificado fue digerido con enzimas de restricción apropiadas EcoRI y NotI (Promega, EEUU) durante $2 \mathrm{~h}$ a $37^{\circ} \mathrm{C}$ y se ligó al vector pET28a (Novagen, EEUU) a $4{ }^{\circ} \mathrm{C}$ durante la noche, de acuerdo con las instrucciones del fabricante. El plásmido recombinante se transformó en $E$. coli BL21(DE3).

\section{Inducción y Purificación de Omp31 Recombinante}

La expresión de la proteína fue inducida en LB suplementado con $30 \mathrm{mg} / \mathrm{ml}$ de kanamicina con la adición $1 \mathrm{mM}$ isopropil-dthiogalactopyranoside (IPTG) y las células transformadas fueron incubadas durante $4 \mathrm{~h}$. Las bacterias fueron recolectadas por centrifugación $\left(12000 \mathrm{~g}\right.$ por $15 \mathrm{~min}$ a $\left.4{ }^{\circ} \mathrm{C}\right) \mathrm{y}$ congeladas a $-20{ }^{\circ} \mathrm{C}$ hasta su uso.

Las células bacterianas se suspendieron en una solución que consiste en Tris 50 mM, EDTA 5 mM, y 1\% de Triton X-100 (pH 8.0) (solución de suspensión) y sonicados durante tres ciclos de $1 \mathrm{~min}$ a $4{ }^{\circ} \mathrm{C}$. Los cuerpos de inclusión fueron recolectados a $15000 \mathrm{~g}$ por $15 \mathrm{~min}$ a $4{ }^{\circ} \mathrm{C}$ y lavadas dos veces con solución de suspensión sin Triton X-100. Los cuerpos de inclusión se solubilizaron en una solución que contiene Tris $50 \mathrm{mM}, 5 \mathrm{mM}$ EDTA y urea $8 \mathrm{M}(\mathrm{pH} 8.0)$ a temperatura ambiente durante la noche con agitación. Después de la centrifugación (15000 g por 15 min a $4{ }^{\circ} \mathrm{C}$ ), la proteína soluble fue purificada mediante cromatografía a través de una resina ProBond (Invitrogen, EEUU). La presencia de Omp31r fue verificado por Western blot con el anticuerpo anti-Omp31 producido en conejo. La pureza fue evaluada mediante electroforesis en gel de SDS-poliacrilamida y tinción con azul de Coomassie.

\section{SDS-PAGE y Ensayos de Western Blot}

EL SDS-PAGE se realizó según Laemmli (Laemmli, 1970). La Omp31r purificada se llevó a $95{ }^{\circ} \mathrm{C}$ durante $10 \mathrm{~min}$ y se diluyó en $2 \mathrm{x}$ tampón de muestra Laemmli (4\% de SDS, $20 \%$ de glicerol, $10 \%$ de $2-$ mercaptoetanol, $0.004 \%$ de azul de bromofenol y $0.125 \mathrm{M}$ de Tris $\mathrm{HCl}, \mathrm{pH} 6.8$ ). Luego de la electroforesis, los geles se tiñeron durante una hora con Azul Brillante de Coomassie (Azul Brillante de Coomassie R250 al $0.1 \%$, ácido acético al $10 \%$, metanol al $50 \%$ ) y posteriormente se destiñeron con una solución de metanol al 50\%. En los ensayos de Wester-blot, después de la electroforesis, la proteína se transfirió a membranas de Immobilon-P (Milipore, EEUU) en tampón de transferencia (Tris $25 \mathrm{mM}$, glicina $192 \mathrm{mM}$ y metanol al 20\%) con una corriente constante de $2 \mathrm{~mA} / \mathrm{cm}^{2}$ durante $30 \mathrm{~min}$, usando un equipo de transferencia semiseco (Bio-Rad, EEUU). La membrana se bloqueó con leche descremada al $5 \%$, durante $1 \mathrm{~h}$ a $4{ }^{\circ} \mathrm{C}$, se lavó tres veces con PBS-Tween 20 al $0.05 \%$ y se incubó con un suero positivo antiOmp31r, producido a partir de un conejo inmunizado con Omp31r, dilución 1: 1.000, en tampón de bloqueo a $4{ }^{\circ} \mathrm{C}$ durante $2 \mathrm{~h}$. La membrana se lavó con $0.05 \%$ de PBS-Tween 20 y se incubó con anticuerpo anti-IgG de conejo hecho en cabra conjugado con peroxidasa de rábano picante (HRP) (dilución 1: 5.000, Sigma, EEUU), en tampón de bloqueo durante $1 \mathrm{~h} \mathrm{a} 4^{\circ} \mathrm{C}$, y finalmente lavado con $0.05 \%$ de PBS-Tween 20 . La proteína se detectó con una solución de detección de diaminobencidina- $\mathrm{H}_{2} \mathrm{O}_{2}$. Los sueros controles positivos a brucelosis de bovinos y negativos fueron donados por el Instituto Nacional de Investigaciones Agrícolas (INIA).

\section{ELISA Usando Omp31r}

Se recubrieron placas de poliestireno (96 pocillos) con Omp31r (0.2 ì/pocillo), diluido en tampón de fosfato solución salina 
(PBS). Los sitios no enlazados en las placas fueron bloqueados con $200 \mu 1$ de PBS que contiene $3 \%$ de albúmina de suero bovino (BSA) por pozo. Posteriormente, los pozos fueron lavados con PBS que contiene $0.05 \%$ de Tween 20 (PBS-T). Los sueros fueron diluidos en PBS-T que contiene $1 \%$ de BSA y colocados en cada pozo.

Se detectaron anticuerpos específicos usando un conjugado anti-bovino peroxidasa. La reacción fue desarrollada agregando TMB ( $2 \mathrm{~g} / \mathrm{l})$ en tampón de $0.1 \mathrm{M}$ citrato-fosfato que contiene $0.03 \%$ de $\mathrm{H}_{2} \mathrm{O}_{2}$. Para establecer los valores de corte del ensayo se usaron sueros controles sanos, no infectados, bajo las mismas condiciones. El valor de corte de cada suero vinculado al sistema de ensayo se calculó como la media de la densidad óptica (DO) específica de los sueros controles más dos desviaciones estándar.

\section{Punto de Corte}

El umbral o punto de corte es el valor de absorbancia (DO) y fue calculado para 32 determinaciones de controles sanos, basados en la relación $\mathrm{X}_{\mathrm{s}}=\mathrm{X}+3 \delta$ (Sutula $\mathrm{y}$ Cuillet, 1986), donde $X_{s=}$ umbral, $X 0$ media de los controles sanos, y $\delta=$ desviación estándar. Esto implica que las muestras pro- blemas que registren una $\mathrm{DO}_{450 \mathrm{~nm}}$ superiores al valor del punto de corte se consideran positivas y los que estén por debajo se consideran como negativas.

\section{Sensibilidad y Especificidad}

La sensibilidad y especificidad de las pruebas se calcularon con respecto a los bovinos infectados y sanos de Brucella, según el método descrito por Hop et al., 2016).

\section{Resultados y Discusión}

El análisis bioinformático de las proteínas Omp31 de B. melitensis y B. abortus mostró un $70 \%$ se similaridad (Figura 1; Cuadro 1). La similitud entre las Omp31 de las mismas especies fue de $100 \%$ (Cuadro 1).

La alta similitud implica que puede existir una reacción cruzada entre ambas proteínas al ser evaluadas con sueros de animales infectados con especies de Brucella que expresen esta proteína. Connolly et al. (2006) detectaron una Omp31 de B. abortus biovar 224 usando sueros de bovinos positivos a brucelosis, por lo que podría encontrarse en otras $B$. abortus silvestres. Es por ello, que esta proteína fue escogida para ser evalua-

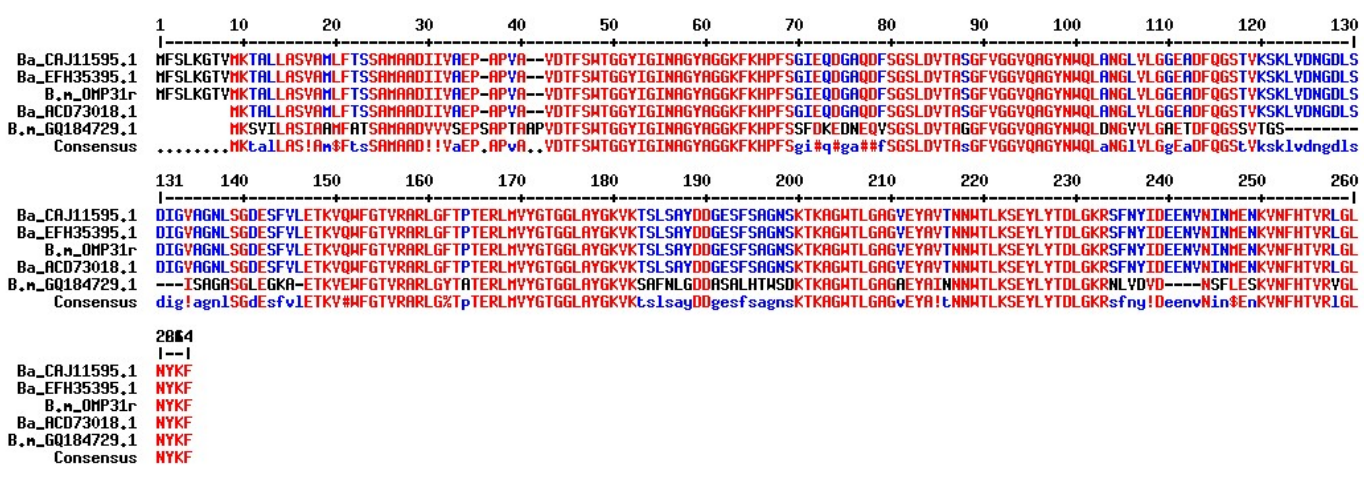

Figura 1. Alineamiento de aminoácidos de proteínas Omp31 usando MultAlin. Alineamiento de la secuencia Omp31r de Brucella melitensis 16M, B.m_OMP31r_GQ184729.1 y otras Brucellas B. abortus 2308, Ba_CAJ11595.1 (GenBank acceso CAJ11595.1), B. abortus B3196, Ba_EFH35395.1 (GenBank acceso EFH35395.1), B. abortus S19, Ba_ACD73018.1, (GenBank acceso. ACD73018), B. melitensis 152 (GenBank acceso GQ184729.1), B.m OMP31r, secuencia obtenida en este trabajo 
Cuadro 1. Porcentaje de identidad de aminoácidos entre diferentes proteínas Omp31

\begin{tabular}{lccccc}
\hline & $\begin{array}{c}\text { B. abortus } \\
2308\end{array}$ & $\begin{array}{c}\text { B. abortus } \\
\text { B3196 }\end{array}$ & $\begin{array}{c}\text { B. abortus } \\
\text { ACD73018 }\end{array}$ & $\begin{array}{c}\text { B. melitensis } \\
152\end{array}$ & $\begin{array}{c}\text { B. melitensis } \\
\text { OMP31r 16M }\end{array}$ \\
\hline B. abortus 2308 & 100 & 100 & 100 & 70 & 100 \\
B. abortus B3196 & 100 & 100 & 100 & 70 & 100 \\
B. abortus ACD73018 & 100 & 100 & 100 & 70 & 100 \\
B. melitensis 152 & 70 & 70 & 70 & 100 & 70 \\
B. melitensis OMP31r & 100 & 100 & 100 & 70 & 100 \\
16M & & & & & \\
\hline
\end{tabular}

da. Se diseñaron oligos para amplificar el gen Omp31 de B. melitensis a partir de ADN genómico (Figura 2A), obteniéndose una banda de $723 \mathrm{pb}$, donde la secuenciación corroboró la identidad del gen (datos no mostrados). Este fragmento fue clonado en el vector de expresión pET28a y fue verificado por PCR (Figura 2B, C, D).

La proteína Omp31r se indujo con $1 \mathrm{mM}$ IPTG y se purificó con cromatografía IMAC (Figura 3). La Omp31r se obtuvo con un rendimiento de $70 \mu \mathrm{g} / \mathrm{ml}$. Esta proteína fue usada para las pruebas de ELISA y Western Blot (Figura 3).

Como antígeno control se utilizó un extracto fenolizado de B. abortus biovar 1119, cepa empleada para la fabricación del Rosa de Bengala (RB). El valor de corte de cada antígeno en la prueba de ELISA (Figura 4) se calculó como la media específica de las DO de 32 sueros libres de Brucella usados como control más dos desviaciones estándar. La media para Omp31r fue de 0.1919 y el punto de corte de 0.3838 . Con el antígeno de Brucella cepa 1119 se obtuvo una media de 0.1183 y un punto de corte de 0.2366 . Los bovinos positivos $(\mathrm{n}=57)$ produjeron un OD entre 0.285 y 0.975 (media: 0.547; D.E.: 0.203). De estos bovinos, se detectaron anticuerpos contra Omp31 en 43 de ellos (75.4\%); y al utilizar la cepa de Brucella 1119 como antígeno los 57 sueros se detectaron como positivos. Al comparar los antígenos, solo 14 sueros positivos no fueron detectados por la Omp31r. La sensibilidad y especificidad de la Omp31r en comparación a la Cepa 1119 fue de $77.2 \%$ y $90.6 \%$, respectivamente.

De los 52 sueros negativos, tres reaccionaron con Omp31r, lo cual pudo deberse a la presencia de proteínas de E. coli que copurificaron en la obtención de la proteína recombinante y dieron reacción cruzada con estos sueros. Esto ha sido reportado para proteínas de membrana Bp26 (Debbarh et al., 1996; Cloeckaert et al., 2001; Liu et al., 2011; Tiwari et al., 2011) y Omp28 (Thavaselvam et al., 2010; Kumar et al., 2012; Lim et al., 2012), empleadas como antígenos en el diagnóstico de brucelosis en ovejas y cabras.

Otra forma de evidenciar estas reacciones cruzadas es realizando un Westernblot, donde al usar sueros de bovinos se observan las bandas contaminantes de E. coli. (Figura 5C). Estas bandas dan reacción cruzada y provocan falsos positivos en ELISA. Esto podría ser mejorado optimizando la purificación de la Omp31r. La especificidad se observa por Western-blot al evaluar sueros positivos, dado que estos reconocen a la banda de Omp31r y una banda de $31 \mathrm{kDa}$ en el extracto de B. abortus 1119 , pero no reconocen a B. abortus RB51 (Figura 5C). Además, sueros de bovinos sanos y vacunados contra B. abortus RB51 no reconocen la banda de Omp31r (Figura 5D, 5E). 


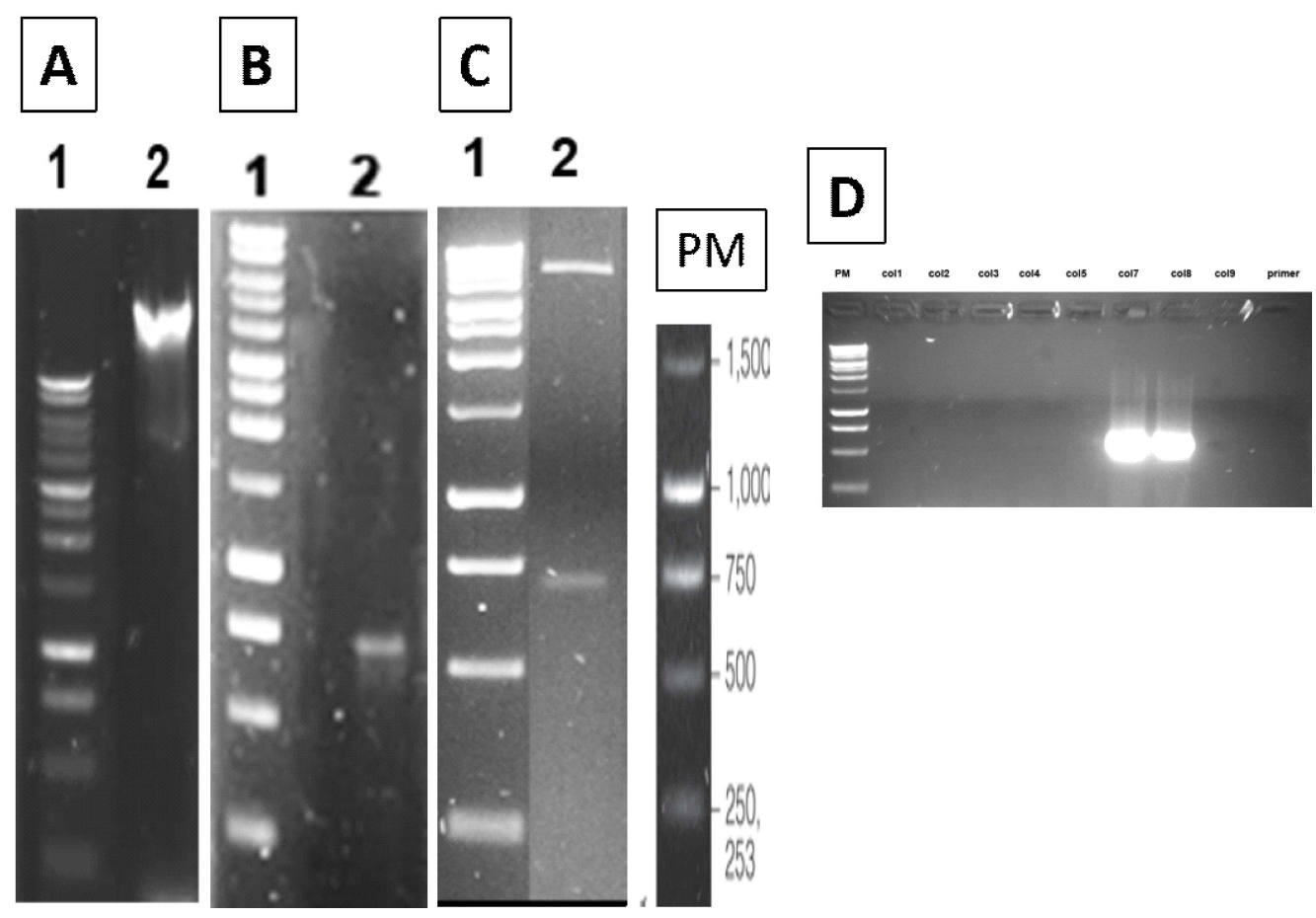

Figura 2. (A) Purificación de ADN genómico de Brucella melitensis 16M; (B) Amplificado del gen Omp31; (C) digestión del vector pET28a con EcoRI/NotI (canal 2); (D) PCRcolonia de diferentes colonias, transformadas con la ligación pET28a/Omp31; (PM) Detalle del marcador de peso molecular de ADN

A

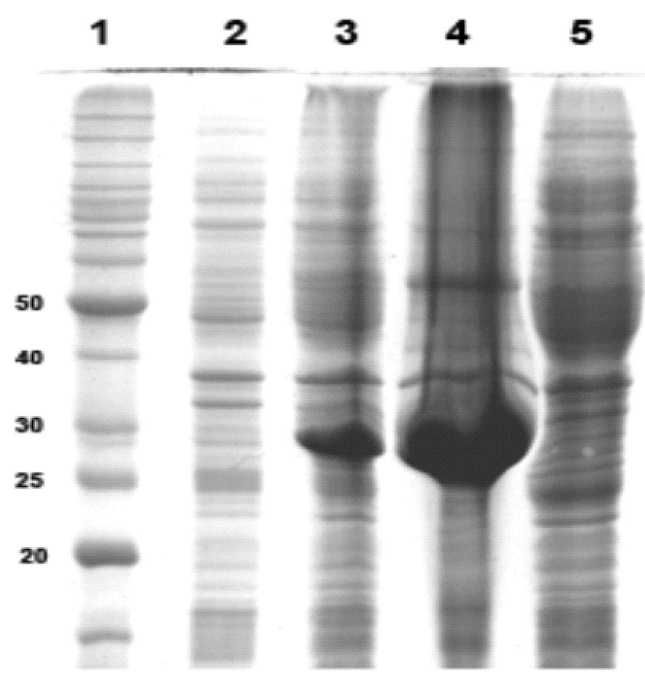

B

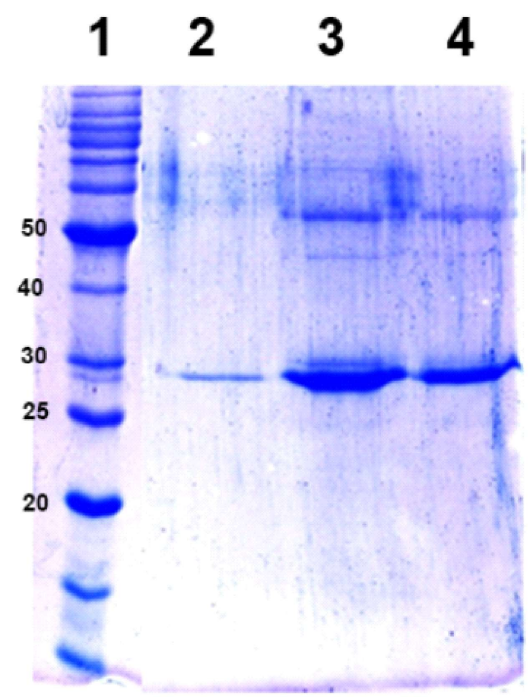

Figura 3. Análisis por SDS-PAGE $12 \%$ de las fracciones obtenidas al inducir la Omp31 recombinante con IPTG a $37^{\circ} \mathrm{C}$. (A): Línea 1, marcadores de peso molecular; línea 2, fracción sin inducir; línea 3, fracción a $3 \mathrm{~h}$ de inducción; línea 4, fracción insoluble, fracción 5, fracción soluble. (B): Línea 1, marcadores de peso molecular; líneas 2 a 4, fracciones eluídas con $250 \mathrm{mM}$ de imidazol. El peso molecular aparente de la Omp31r es de $28 \mathrm{kDA}$ 


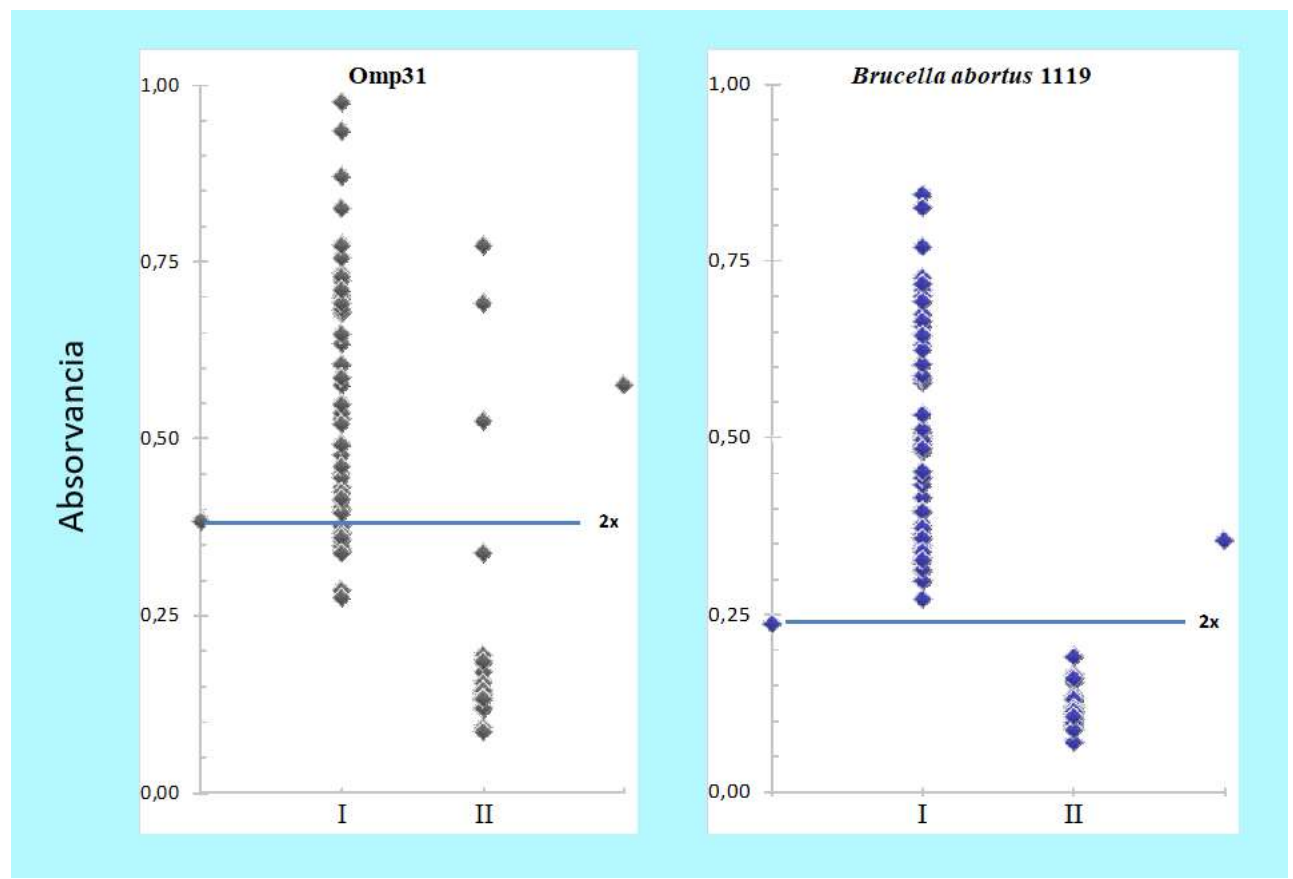

Figura 4. Datos de reactividad del antígeno recombinante Omp31r empleado en el serodiagnóstico por ensayo de ELISA. Se evaluó la Omp31r (20 ng/pozo) y un extracto de Brucella abortus biovar 1119 ( $2 \mathrm{ng} /$ pozo) usando 82 sueros de bovinos clasificados por grupos: I, sueros positivos a brucelosis y II, sueros bovinos negativos a brucelosis. La línea gruesa horizontal indica el valor del punto de corte para cada antígeno

Este resultado es importante ya que discrimina animales no vacunados de los vacunados, por lo que se evidencia su potencial en el desarrollo de pruebas diagnósticas de brucelosis. Entre las pruebas convencionales está Rosa de Bengala (RB) (RamirezPfeiffer et al., 2008) y la fijación del complemento (FC) (Blasco et al., 1994; Adone y Ciuchini, 2001), así como las pruebas que usan extractos ricos en S-LPS (como antígeno y usando un anticuerpo monoclonal) (Chin et al., 1989; Laurent et al., 2004; Nielsen et al., 2007; Perrett et al., 2010). Estas pruebas tienen en común que detectan principalmente anticuerpos antigénicos determinantes, presentes en la cadena O del LPS-S (Dzata et al., 1991). En el presente trabajo, el potencial diagnóstico de la Omp31r muestra que el formato ELISA es útil para el diagnóstico de infección por B. abortus en bovinos; sin em- bargo, el uso de Omp31 no ha sido reportado para su uso en bovinos, sino para cabras y ovejas (Cassataro et al., 2004; Jacques et al., 2007; Estein et al., 2009). Esto se debe a que $B$. melitensis infecta a estos animales de manera específica; sin embargo, al no encontrarse la proteína en $B$. abortus no había sido usada en bovinos (Vizcaino et al., 2001).

Por otro lado, se ha probado extractos de $B$. abortus por ELISA que resultan más sensibles que la Omp31r, por lo que en este trabajo se usó a $B$. abortus 1119. La baja sensibilidad de Omp31r podría deberse a que el bovino no desarrolla anticuerpos específicos contra esta o, desde el punto de vista técnico, la Omp31r no se unió adecuadamente a la placa de ELISA por ser hidrofóbica. No se puede descartar que la ausencia de reactividad de anticuerpos contra esta pro- 


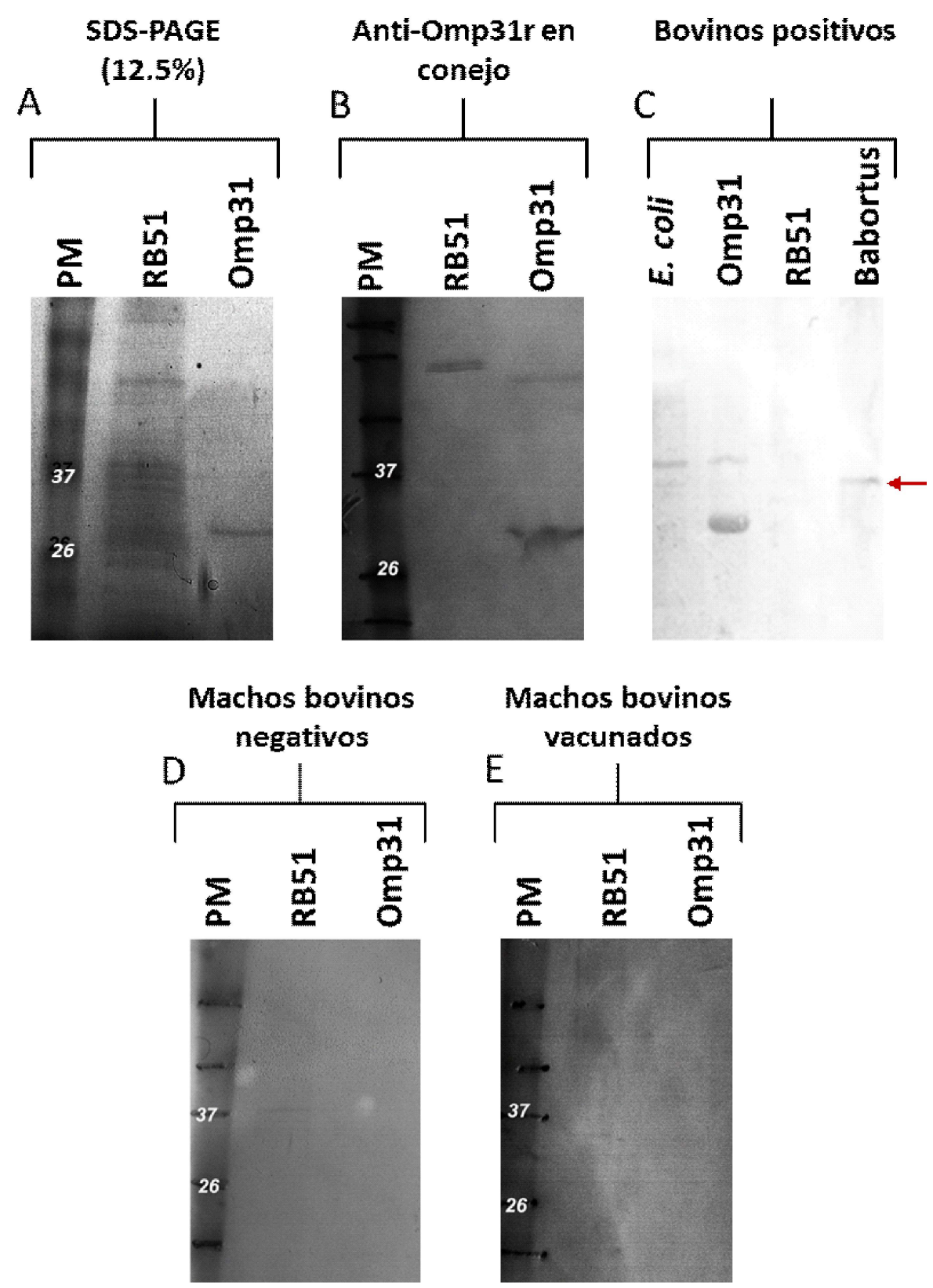

Figura 5. Western-Blot evaluando un extracto de E. coli, Omp31r (10 $\mu \mathrm{g} / \mathrm{pozo})$, un extracto de B. abortus RB51 y un extracto de $B$. abortus cepa 1119 por anticuerpos específicos para Omp31r y sueros bovinos sanos y positivos a brucelosis. (A) Las proteínas (10 $\mu \mathrm{g}$ de cada fracción) fueron separadas en SDS-PAGE (12.5\%) y teñidas con azul de coomassie. (B) Fueron electroforéticamente transferidas a nitrocelulosa y detectadas con anti-Omp31 $1_{\mathrm{R}}$ realizado en conejo (dilución $1: 1000$ ) y conjugado (1:8000). Se cargó $0.75 \mu \mathrm{g}$ de Omp31r. Las posiciones de los marcadores de peso molecular son indicadas a la izquierda. (C) Se usaron tres sueros bovinos positivos que reconocen la banda de Omp31r y la banda de Omp31 nativa en el extracto de B.abortus 1119 (reconocen bandas en el extracto de E. coli, pero no reconoce a B. abortus RB51). (D) Al usar una mezcla de tres sueros negativos de machos bovinos, no reconocen la banda de Omp31. (E) Al usar tres sueros de machos bovinos vacunados con la vacuna RB51 se observa reconocimiento de la banda de Omp31. Los sueros se usaron a una dilución 1:100 y el conjugado a 1:5000 
teína también pudiera deberse a la eliminación de epítopos conformacionales, debido a la desnaturalización de la proteína bajo condiciones que se realizó la prueba de ELISA.

Al realizar los ensayos de ELISA ( $F i-$ gura 4), los valores de sensibilidad (77.2\%) y especificidad $(90.6 \%)$ encontrados para Omp31r en bovinos son similares a los reportados en ovejas, cabras, y humanos evaluados con las proteínas de membrana recombinantes Bp26 (Debbarh et al., 1996; Cloeckaert et al., 2001; Tiwari et al., 2011) con sensibilidad y especificidad de 88 y $93 \%$, respectivamente, para Omp 28 de $97.5 \%$ de sensibilidad y $85.9 \%$ de especificidad (Thavaselvam et al., 2010) y para Omp31 de $67 \%$ de sensibilidad (Cassataro et al., 2004).

\section{ConClusiones}

A partir de ADN genómico de Brucella melitensis se logró clonar, expresar y purificar la proteína recombinante Omp31, la cual presentó una alta sensibilidad (77.2\%) y especificidad $(90.6 \%)$ en el diagnóstico por ELISA de sueros bovinos.

\section{Agradecimiento}

Los autores agradecen al Dr. De Sanctis JB por sus valiosos aportes al manuscrito.

\section{Literatura Citada}

1. Adone R, Ciuchini F. 2001. Brucella abortus RB51 and hot saline extract from Brucella ovis as antigens in a complement fixation test used to detect sheep vaccinated with Brucella abortus RB51. Clin Diagn Lab Immunol 8: 119122. doi: 10.1128/CDLI.8.1.119. 122.2001

2. Al Dahouk S, Tomaso H, Nockler K, Neubauer H, Frangoulidis D. 2003. Laboratory-based diagnosis of brucellosis - a review of the literature. Part I:
Techniques for direct detection and identification of Brucella spp. Clin Lab 49: 487-505.

3. Blasco JM, Garin-Bastuji B, Marin CM, Gerbier G, Fanlo J, Jimenez de Bagues MP, Cau C. 1994. Efficacy of different Rose Bengal and complement fixation antigens for the diagnosis of Brucella melitensis infection in sheep and goats. Vet Rec 134: 415-420.

4. Bricker BJ. 2002. Diagnostic strategies used for the identification of Brucella. Vet Microbiol 90: 433-434.

5. Bundle DR, Gidney MA, Perry MB, Duncan JR, Cherwonogrodzky JW. 1984. Serological confirmation of Brucella abortus and Yersinia enterocolitica O:9 O-antigens by monoclonal antibodies. Infect Immun 46: 389-393.

6. Cassataro J, Pasquevich K, Bruno L, Wallach JC, Fossati CA, Baldi PC. 2004. Antibody reactivity to Omp31 from Brucella melitensis in human and animal infections by smooth and rough Brucellae. Clin Diagn Lab Immunol 11: 111-114. doi: 10.1128/CDLI.11.1.111114.2004

7. Chavez-Fumagalli MA, Schneider MS, Lage DP, Machado-de-Avila RA. Coelho EA. 2017. An in silico functional annotation and screening of potential drug targets derived from Leishmania spp hypothetical proteins identified by immunoproteomics. Exp Parasitol 176: 66-74. doi: 10.1016/j.exppara.2017.03.005

8. Chenais E, Bagge E, Lambertz ST, Artursson K. 2012. Yersinia enterocolitica serotype O:9 cultured from Swedish sheep showing serologically false-positive reactions for Brucella melitensis. Infect Ecol Epidemiol 2. doi: 10.3402/iee.v2i0.19027

9. Chenchev I, Khristoforov L, Peshkov I, Kostov G, Mineva I. 1978. [Diagnostic value of agglutination reaction with buffered Brucella antigen stained with rose bengal]. Vet Med Nauki 15: 3-9. 
10. Chin J, Daniels J, Bundesen P. 1989. Bovine brucellosis: evaluation of field sera by a competitive and superimposable ELISA utilising a monoclonal antibody against Brucella abortus lipopolysaccharide. Vet Immunol Immunopathol 20: 109-118. doi: 10.1016/ 0165-2427(89)90092-5

11. Cloeckaert A, Baucheron S, Vizcaino $N$, Zygmunt MS. 2001. Use of recombinant BP26 protein in serological diagnosis of Brucella melitensis infection in sheep. Clin Diagn Lab Immunol 8: 772-775. doi: 10.1128/ CDLI.8.4.772-775.2001

12. Connolly JP, Comerci D, Alefantis TG, Walz A, Quan M, Chafin R, Grewal P, et al. 2006. Proteomic analysis of Brucella abortus cell envelope and identification of immunogenic candidate proteins for vaccine development. Proteomics 6: 3767-3780. doi: 10.1002/ pmic. 200500730

13. Debbarh HS, Zygmunt MS, Dubray G, Cloeckaert A. 1996. Competitive enzyme-linked immunosorbent assay using monoclonal antibodies to the Brucella melitensis BP26 protein to evaluate antibody responses in infected and $B$. melitensis Rev.1 vaccinated sheep. Vet Microbiol 53: 325-337.

14. Dzata GK, Wyckoff III JH, Confer $A W .1991$. Immunopotentiation of cattle vaccinated with a soluble Brucella abortus antigen with low LPS content: an analysis of cellular and humoral immune responses. Vet Microbiol 29: 1526. doi: 10.1016/0378-1135(91)90107-Q

15. Estein SM, Cheves PC, Fiorentino MA, Cassataro J, Paolicchi FA, Bowden RA. 2004. Immunogenicity of recombinant Omp31 from Brucella melitensis in rams and serum bactericidal activity against $B$. ovis. Vet Microbiol 102: 203-213. doi: 10.1016/j.vetmic.2004.05.004

16. Estein SM, Fiorentino MA, Paolicchi FA, Clausse M, Manazza J, Cassataro J, Giambartolomei GH, et al. 2009. The polymeric antigen BLSOmp31 confers protection against Brucella ovis infection in rams. Vaccine 27: 6704-6711. doi: 10.1016/j.vaccine.2009.08.097

17. Francisco J, Vargas O. 2002. Brucellosis in Venezuela. Vet Microbiol 90: 39-44. doi: 10.1016/S03781135(02)00243-2

18. Goldbaum FA, Velikovsky CA, Baldi PC, Mortl S, Bacher A, Fossati CA. 1999. The $18-\mathrm{kDa}$ cytoplasmic protein of Brucella species - an antigen useful for diagnosis - is a lumazine synthase. $\mathrm{J}$ Med Microbiol 48: 833-839. doi: 10.1099/ 00222615-48-9-833

19. Hu DD, Cui J, Xiao D, Wang L, Liu $L N$, Liu RD, Zhang JZ, Wang $Z Q$. 2014. Identification of early diagnostic antigens from Spirometra erinaceieuropaei sparganum soluble proteins using immunoproteomics. Southeast Asian J Trop Med Public Health 45: 576-583.

20. Jacques I, Verger JM, Laroucau K, Grayon M, Vizcaino $N$, Peix A, Cortade F, et al. 2007. Immunological responses and protective efficacy against Brucella melitensis induced by bp 26 and omp31 B. melitensis Rev. 1 deletion mutants in sheep. Vaccine 25: 794-805. doi: 10.1016/j.vaccine.2006.09.051

21. Kim JY, Sung SR, Lee K, Lee HK, Kang SI, Lee JJ, Jung SC, et al. 2014. Immunoproteomics of Brucella abortus RB51 as candidate antigens in serological diagnosis of brucellosis. Vet Immunol Immunopathol 160: 218-224. doi: 10.1016/j.vetimm.2014.05.009

22. Kumar A, Tiwari S, Thavaselvam D, Sathyaseelan K, Prakash A, Barua A, Arora S, Kameswara RM. 2012. Optimization and efficient purification of recombinant Omp28 protein of Brucella melitensis using Triton X-100 and betamercaptoethanol. Protein Expr Purif 83: 226-232. doi: 10.1016/j.pep.2012.04.002

23. Laemmli UK. 1970. Cleavage of structural proteins during the assembly of the head of bacteriophage T4. Nature 227: 680-685. 
24. Laurent TC, Mertens P, Dierick JF, Letesson JJ, Lambert C, De Bolle X. 2004. Functional, molecular and structural characterisation of five antiBrucella LPS mAb. Mol Immunol 40: 1237-1247. doi: 10.1016/j.molimm.2003.11.037

25. Lim JJ, Kim DH, Lee JJ, Kim DG, Min W, Lee HJ, Rhee MH, Kim S. 2012. Protective effects of recombinant Brucella abortus Omp28 against infection with a virulent strain of Brucella abortus 544 in mice. J Vet Sci 13: 287-292. doi: 10.4142/jvs.2012.13.3.287

26. Liu WX, Hu S, Qiao ZJ, Chen WY, Liu LT, Wang FK, Hua RH, et al. 2011. Expression, purification, and improved antigenic specificity of a truncated recombinant bp26 protein of Brucella melitensis M5-90: a potential antigen for differential serodiagnosis of brucellosis in sheep and goats. Biotechnol Appl Biochem 58: 32-38.

27. Lord V, Flores R. 1983. Brucella $\mathrm{spp}$ from the capybara (Hydrochaeris hydrochaeris) in Venezuela: serologic studies and metabolic characterization of isolates. J Wildl Dis 19: 308-314. doi: 10.7589/0090-3558-19.4.308

28. Lord V, Lord R. 1991. Brucella suis infections in collared peccaries in Venezuela. J Wildl Dis 27: 477-481. doi: 10.7589/0090-3558-27.3.477

29. Nielsen K. 2002. Diagnosis of brucellosis by serology. Vet Microbiol 90: 447-459. doi: /10.1016/S0378-1135(02)00229-8

30. Nielsen K, Smith P, Yu WL, Elmgren C, Nicoletti P, Perez B, Bermudez R, Renteria T. 2007. Second generation competitive enzyme immunoassay for detection of bovine antibody to Brucella abortus. Vet Microbiol 124: 173-177. doi: 10.1016/j.vetmic.2007.03.023

31. Pellon A, Ramírez-García A, Buldain I, Antoran A, Rementeria A, Hernando FL. 2016. Immunoproteomics-based analysis of the immunocompetent serological response to
Lomentospora prolificans. J Proteome Res 15: 595-607. doi: 10.1021/acs.jproteome.5b00978

32. Perret LL, McGiven JA, Brew SD, Stack JA. 2010. Evaluation of competitive ELISA for detection of antibodies to Brucella infection in domestic animals. Croat Med J 51: 314-319.

33. Ramírez-Pfeiffer C, Díaz-Aparicio E, Gómez-Flores R, Rodríguez-Padilla C, Morales-Loredo A, Alvarez-Ojeda G. 2008. Use of the Brucella melitensis native hapten to diagnose brucellosis in goats by a rapid, simple, and specific fluorescence polarization assay. Clin Vaccine Immunol 15: 911-915. doi: 10.1128/CVI.00046-08

34. Tan W, Wang XR, Nie Y, Wang C, Cheng $L Q$, Wang $X C$, Zhang $R$, Yan GM. 2012. Recombinant VirB5 protein as a potential serological marker for the diagnosis of bovine brucellosis. Mol Cell Probes 26: 127-131.

35. Thavaselvam D, Kumar A, Tiwari S, Mishra M, Prakash A. 2010. Cloning and expression of the immunoreactive Brucella melitensis $28 \mathrm{kDa}$ outermembrane protein (Omp28) encoding gene and evaluation of the potential of Omp28 for clinical diagnosis of brucellosis. J Med Microbiol 59: 421-428. doi: 10.1099/jmm.0.017566-0

36. Tiwari AK, Kumar S, Pal V, Bhardwaj B, Rai GP. 2011. Evaluation of the recombinant 10-kilodalton immunodominant region of the BP2 6 protein of Brucella abortus for specific diagnosis of bovine brucellosis. Clin Vaccine Immunol 18: 1760-1764. doi: 10.1128/ CVI.05159-11

37. Tjalsma H, Schaeps RM, Swinkels $D W$. 2008. Immunoproteomics: from biomar-ker discovery to diagnostic applications. Proteomics Clin Appl 2: 167-180. doi: 10.1002/prca.200780012

38. Vizcaino N, Cloeckaert A, Zygmunt MS, Dubray G. 1996. Cloning, nucleotide sequence, and expression of the Brucella melitensis omp31 gene 
coding for an immunogenic major outer membrane protein. Infect Immun 64: 3744-3751.

39. Vizcaino N, Kittelberger R, Cloeckaert A, Marin CM, Fernandez-Lago L. 2001. Minor nucleotide substitutions in the omp31 gene of Brucella ovis result in antigenic differences in the major outer membrane protein that it encodes compared to those of the other Brucella species. Infect Immun 69: 7020-7028. doi: 10.1128/IAI.69.11.7020-7028.2001

40. Zhao Z, Yan F, Ji W, Luo D, Liu X, Xing L, Duan Y, et al. 2011. Identification of immunoreactive proteins of Brucella melitensis by immunoproteomics. Sci China Life Sci 54: 880-887. doi: 10.1007/s11427-011-4218-2 